\title{
The use of APL in teaching multivariate data analysis
}

\author{
LELAND WILKINSON and L. ROWELL HUESMANN \\ Yale University. New Haven, Connecticut 06510
}

APL is recommended for introducing students in multivariate data analysis to computer applications of statistical formulas. Limitations and advantages of the language for this purpose are discussed, and examples of basic operations and statistical analyses are presented.

The "behold" proof, using sample data for elucidating statistical procedures, has been particularly useful for students of applied statistics. Working through numerical examples, psychology students in courses on statistical inference have learned not only how to analyze research data, but also why particular analyses are variously appropriate.

Two approaches to teaching multivariate data analysis are now prevalent. On the one hand, calculators have been used on problem sets involving simplified data. But students must spend considerable time learning computational formulas designed for efficient use of calculators. Moreover, these formulas usually obscure the functions underlying the computations. Finally, calculator operations soon become tedious for the student. On the other hand, "canned" computer programs have been used on problem sets such as those in Morrison (1967) and Tatsuoka (1972). In this case, students' familiarity with procedures is limited to the textbook, and the computer's function is opaque. Cooley and Lohnes (1971) have included a chapter on FORTRAN programming and multivariate program listings to correct this problem, but students must spend considerable effort in learning the language before they can get to applications.

These two approaches leave room for a third: interactive multivariate analysis. APL allows direct manipulations by the student of his data matrices according to the matrix formulas found in most texts. Unlike calculators and "canned" programs, APL provides a transparent medium for developing data analysis from theoretical statistics.

A number of features of APL make it ideally suited for interactive, instructional data analysis. It is first necessary to understand its limitations, however. First of all, IBM's APL software package defines a workspace size of $32 \mathrm{~K}$ bytes. Since dynamic APL program allocation is not included, this means that programs and data can occupy no more than a fixed region of $32 \mathrm{~K}$ bytes in core. Real numbers occupy 8 bytes, integers 4 . so a 64 by 64 data matrix can more than fill up a workspace. Second, systems utilities are limited in API to terminal I/O, unless the IBM 1050 terminal, which interfaces with paper tape and card reader, is used in place of the 2741. Third. APL is interpretive. so executions involving steps repeated a large number of times can cause considerable compilation. None of these limitations inhere in the language design, of course. Various installations have overcome some or all of them, but IBM support presently accompanies only the standard package.

These limitations do not affect the usefulness of APL as an instructional language for multivariate statistics, however. No special programming or hardware is necessary for making an APL system immediately applicable to a classroom curriculum.

First of all, the real-time environment of APL lends itself to such use. Students can begin immediately on problems without an introduction to computing. Error messages are clear, and usually direct proper reentry. Most important, the language is operationally intact; students need not learn the entire language structure before active use. Nearly always, what users don't know won't hurt them, although deeper knowledge enables simpler solutions. Nevertheless, this high level of machine independence has not prevented APL from being used as a means for introducing systems (Katzan, 1970).

Second, APL is in itself upward compatible. From simple scalar desk-calculator operations, students can move to array manipulations, function programming, and execution of statistical functions. Debugging is aided by APL's linear compilation. Programs can be restarted after editing faulty lines.

Third, because its development was motivated by an interest in unified mathematical notation (Iverson, 1962), APL generalizes to matrix operations without alterations in syntax. APL symbols do not obscure matrix formulations. Iterative loops are usually avoidable. Powerful indexing, scanning, and ordering operators exist for row, column, and dimensional manipulation. In addition, single symbols exist for trigonometric functions, logarithms, combinations, permutations. factorials, random number generation, numerical base value, and Boolean operations. The several language manuals available clearly develop their use (Falkoff \& Iverson, 1968; Gilman \& Rose, 1970; Katzan, 1971).

Figure 1 illustrates basic APL usage in desk-calculator mode. User entries are indented. Many of the symbols are taken from ordinary mathematical notation.

The first example shows assignment (the left arrow is equivalent to the FORTRAN =, and the $\mathrm{APL}=$ is like the FORTRAN .EQ.). An assigned variable can be used in subsequent expressions. as shown. Two types of minus sign are indicated. The high bar applies to constants and makes only its adjacent constant negative. The low bar negates everything to its right, as would an ordinary minus sign before a parenthesized expression. 


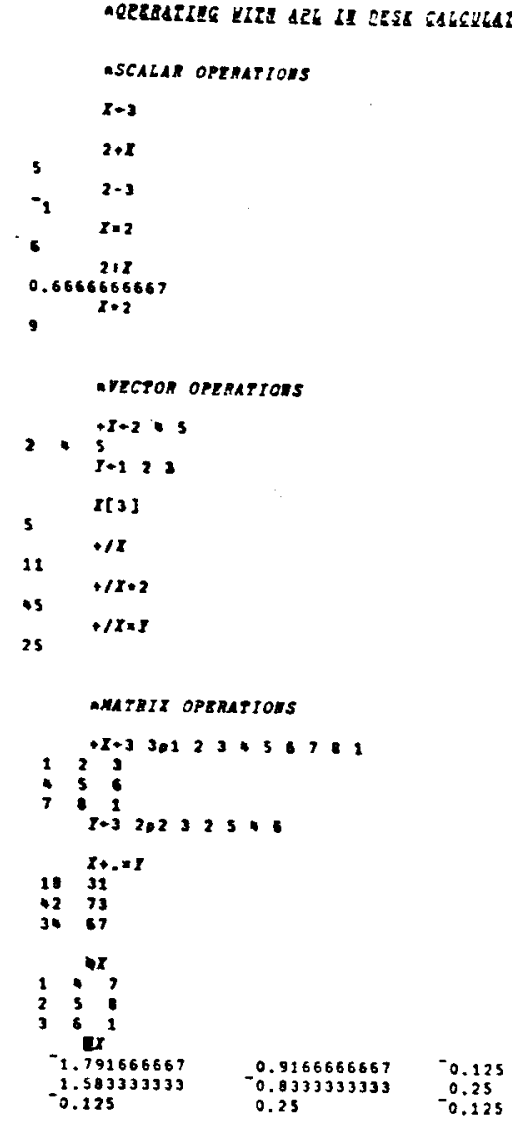

Fig. 1. Sample scalar, vector, and matrix operations with APL in desk-calculator mode. User entries are indented, computer replies are at the margin.

Times and divide resemble ordinary notation, while power is represented by *.

Specification is no different for vectors. They may be indexed as shown. The $+/$ operation is called "plus reduction." It sums the vector elements. Its more general form is $f / X$, where $f$ is any primitive function symbol and $X$ is the vector. The operation interposes the symbol $f$ between all adjacent elements of $X$. The following examples show how the method can be used to obtain sums of squares and cross products, respectively.

Matrix specification is shown together with dimensioning, performed by the rho symbol. The vector specified to $X$ is simultaneously shaped into a 3 by 3 array, creating row and column coordinates. Matrix multiplication is shown next. The general form for this operation is $X f . g Y$, where $f$ and $g$ are primitive function symbols and $X$ and $Y$ are conformable matrices. The $g$ function is first performed between corresponding row and column elements of $X$ and $Y$, respectively, and then the $f$ function is interposed between adjacent elements of the resulting vector. The final result for all rows and columns follows the dimensional rules for matrix conformability. One symbol each performs matrix transpose and inversion, respectively. Transposition can be done on any axis of the matrix in a similar manner.
Figure 2 contains various multivariate analyses on sample data. Students in a course on multivariate analysis taught by the second author have been assigned similar problems for solution using APL in desk-calculator mode. These examples are not solved using the simplest expressions in every case. Extra parentheses and specific notations have been selected to increase the correspondence between the APL evaluations and the matrix equations. The data matrices have been entered previously and are listed. The data consist of four scores on a Rorschach test by two groups of Ss: one a group of parents of schizophrenics and the other a group of control Ss (Morrison, 1968, p. 155).

We begin our analysis by computing basic statistics and intercorrelations. Column totals are derived by plus reduction over the first coordinate, i.e., rows. Element by element division by $\mathrm{N}$ ( 10 and 6 , respectively) produces the means. The variance-covariance matrix obtained is a biased estimate, since the $A$ matrix had $N=10$ in its element denominators. The divide symbol is used monadically in creating the A matrix. That is, when no value appears immediately to its left (dyadically), it creates the reciprocal of the value to its right. Division by 10 for the CXXS matrix is dyadic, and is performed element by element throughout the matrix. To compute the correlation matrix, we use an identity matrix. This is specified by filling a 4 by 4 matrix with repetitions of $\left(\begin{array}{lllll}1 & 0 & 0 & 0 & 0\end{array}\right)$ by rows. The DS matrix is formed by inverting the diagonal matrix produced through element-by-element multiplication of the square root of CXXS.

Given the intercorrelations, we go on to compute a regression equation for predicting one score from the others. The regression computation involves partitioning the variance-covariance matrix through matrix indexing. The ; in APL is the equivalent of the FORTRAN , for indexing. Elements selected in CXXS are subscripted by all combinations of the two vectors (2 34 ) and (2 34). The compilation of the multiple correlation coefficient shows a further use of indexing. The square root could be taken on the same line as the computation, with parentheses around the entire $R^{2}$ quantity. We also compute a pooled variance-covariance matrix, using weights of $\mathrm{N}=10$ to correspond to the biased $\mathrm{N}$ used in their calculation. Multiplication and division are, of course, element by element.

Next, we show a linear discriminant analysis. The computation of Hotelling's $\mathrm{T}^{2}$ and the linear discriminant function require no new procedures from those preceding. The linear discriminant function is for the raw scores data matrices.

Finally, we present a histogram for the SCHIZ matrix plotted by a function named HIST. ${ }^{1}$ This function takes an explicit argument (SCHIZ) analogous to the FORTRAN function subroutine. APL's wealth of characters allows diverse plotting graphics. IBM supplies 
anactses or roun vaplazes on sent2omermic

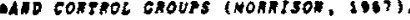

sents

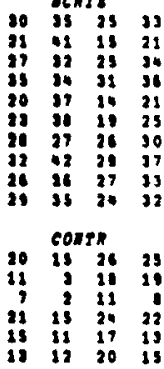

18 12 20 is

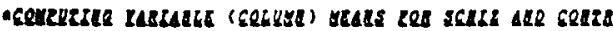

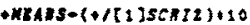

21.235 .723 .530 .2

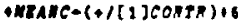

16.5 1.68686667 $19.33333333 \quad 17$

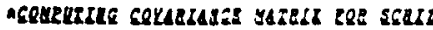

aLE TEOATA MATRIX

ALET A=QUADRATTC PORN COEPFICTERTS MATAIX

weF MenUmese of OAservations

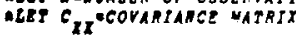

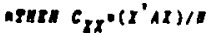

$1-(1010) 0(2-110), 100-110$

\begin{tabular}{|c|c|c|}
\hline $\begin{array}{l}20.49 \\
2 . .97 \\
22.75 \\
22.98\end{array}$ & 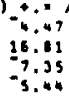 & $\begin{array}{r}+. \quad 5(n I 2)+10 \\
22.75 \\
=7.35 \\
29.25 \\
29\end{array}$ \\
\hline
\end{tabular}

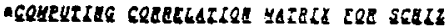

aLT T=IOENTITY MATRIX

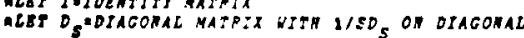

ILET REX EORPELATION NATEIX

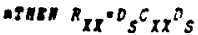

$1+4 \cdot 0(1,4,0)$

DSATI I CXXs) 10.S

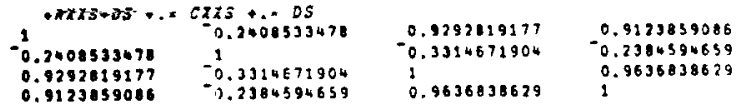

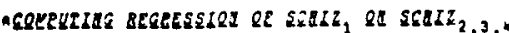

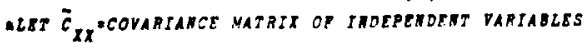

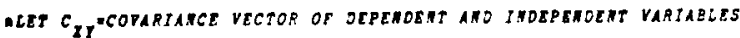

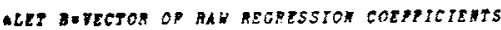

athen $B=\bar{c}_{x x}{ }^{-2} c_{x y}$

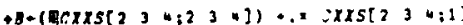

0.068446827540 .63999230000 .1368503238

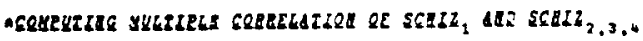
$A R^{2}=\left(C_{X I}, B\right) / \operatorname{VAR}(\mathrm{I})$

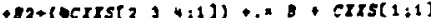

0.1304383582

$\because A+(R 2)=, 3$

0.9329120604

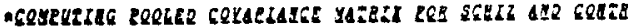

aLET $c_{X X 1}$ cCOARTA.VE MATRIX FOR FRST GROUP

ater $c_{X X 2}$-COVAPIALCE YATAIX FOR SECORD GROUP

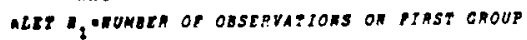

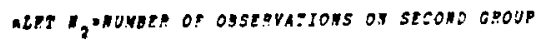

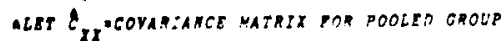

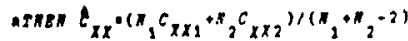

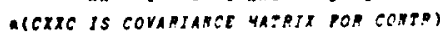

+exxp+(10-cxxs) + (6.exxe)), (10.6-2)

$24.61571429 \quad 6.95 \quad 29.6789143$

$6.35 \quad 23.95952361 \quad 4.154761905$

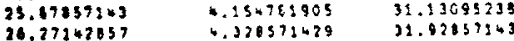

26.27162157

432191429

35.971 .3057

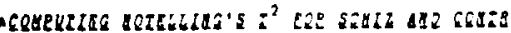

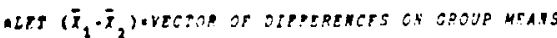

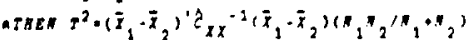

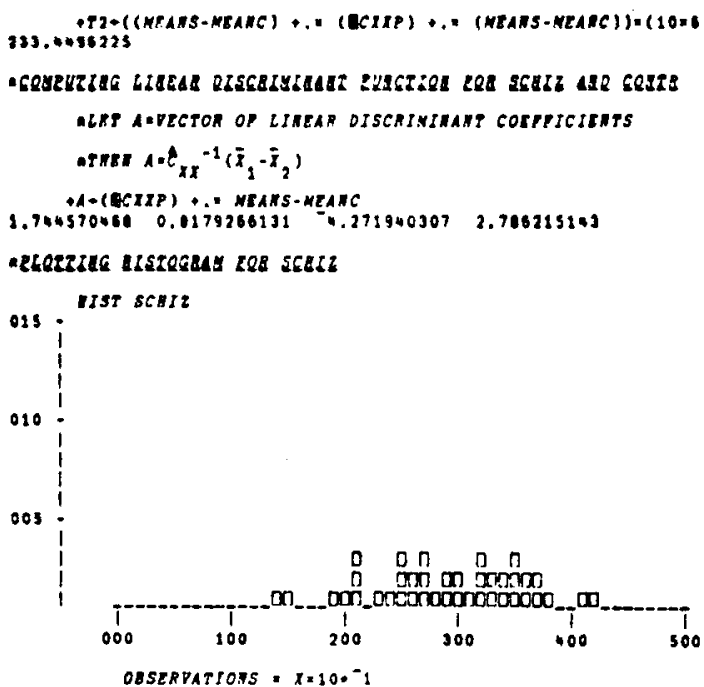

Fig. 2. Sample multivariate analyses in APL on data from Morrison $(1967$, p. 155).

a more general plotting routine called PLOT in its system library of public work spaces.

\section{CONCLUSION}

In these simple calculations, APL is shown to be both clear and concise. Students are stimulated to experiment with actual data interactively. The characteristics of the language itself-extreme machine independence, structural upward compatibility, and generality to matrix operations-make it especially useful for interactive multivariate data analysis. In addition, although we have not given examples, students can write and call functions for other procedures such as principal components and factor analysis (Armitage, 1971). Once introduced to data manipulation with classroom work in theoretical derivations, students are better prepared to handle packaged programs wisely, and can save time and money analyzing smaller data sets interactively.

\section{REFERENCES}

Armitage, E. G. APL: Public library documentation manual. Edmonton: University of Alberta, 1971

Cooley, W., \& Lohnes, P. Multivariate data analysis. New York: Wiley, 1971.

Falkoff, A. D., \& Iverson, K. E. APL $/ 360$ user's manual. (2nd ed.) Yorktown Heights, N.Y: T. J. Watson Research Center, IBM, 1968 (GH 20-0683).

Gilman, L., \& Rose, A. J. APL/360: An interoctive approach. New York: Whley, 1970

Iverson, K. E. A programming language. New York: Wiley, 1962.

Katzan, H. APL programming and computer techniques. New York: Van Nostrand Reinhold, 1970.

Katzan. H, APL user's guide. New York: Van Nostrand Reinhold, 1971

Morrison, D. F. Multivariate statistical methods. New York: McGraw-Hill, 1967

Tatsuoka, M. Multivariote analy'sis. New York: Wiley, 1972.

NOTE

1. This function was written by Gerard Dallal, Department of Statistics, Yale University. 